\title{
The surprising blindness in modern psychiatry: do guidelines really guide?
}

\author{
Pedro Antônio Schmidt do Prado-Lima*
}

BraIns (Brain Institute, PUCRS) InsCer (Instituto do Cérebro, PUCRS), Porto Alegre, Rio Grande do Sul, Brazil

First published online 21 November 2016

There is a surprising blindness in modern psychiatry: do guidelines really guide?

I am a clinical psychiatrist, and as such I struggle to provide my patients with the best treatment. Since my early days as a doctor, even during my training as a psychiatrist, I have worked with treatment-resistant patients, that is, patients for whom the standard treatments recommended in the guidelines often prove useless. These patients' existence and suffering are real, and as a professional dedicated to help such patients improve, I have to find some solution. So, what should be done?

I have no single or final answer to this question. But I have given it considerable thought, and I believe the answer lies, at least in part, in the way we deal with the evidence that forms the basis of the guidelines.

Of course, evidence must be our guiding light, and the rise of the concept of evidence-based medicine changed the face of medical practice, making it more scientific. In other words, a belief-based or prescription culture has been replaced by treatment based on information provided by scientific experimentation, which has improved the quality of the treatments created since then. Nonetheless, are we really in fact using the scientific method? I don't believe so.

I remember a classic turn of phrase used in a statistics course I attended when I was a young psychiatrist: "Compare apples with apples and oranges with oranges. Do not compare apples with oranges." Classic indeed! But this very elementary lesson seems to have been partially forgotten in the case of the evidence produced in psychopharmacology. I make this statement based on the fact that in the vast majority of cases, the patients selected for psychopharmacological studies are very different from the patients that we treat in our offices and hospitals.

* Address for correspondence: Pedro Antônio Schmidt do Prado-Lima, MD, PhD, BraIns (Brain Institute, PUCRS), Av Ipiranga, 6690, prédio 63, Porto Alegre, Rio Grande do Sul, Brazil, Zip Code 90610-000.

(Emails: mailto:paspl@uol.com.br; paspl@uol.com.br)
Let's start with the typical patient selected for a psychopharmacological study. The objective is to select a homogeneous group of patients suffering from a given disorder in order to ensure, after random assignment, with the minimum number of subjects, that we have 2 very similar groups. With these 2 very homogenous groups, we can compare 2 different interventions, one in each group: in general, placebo versus a medication, or an old versus a new medication. To maintain the rule of comparing apples with apples, and not with oranges, both groups must be homogeneous. How can we obtain such homogeneity?

The first step is to avoid comorbidities that could change the response to our intervention. However, in fact, comorbidities are very common in psychiatry. For example, bipolar disorder shows high comorbidity with panic, generalized anxiety, post-traumatic, obsessive compulsive and attention deficit and hyperactivity disorders, and vice-versa. Indeed, $59 \%$ of patients with bipolar II disorder and $55.4 \%$ of patients with bipolar I disorder were also diagnosed with some other form of anxiety disorder according to the Diagnostic and Statistical Manual of Mental Disorders, Fourth Edition (DSM-IV) criteria. ${ }^{1}$ Nevertheless, patients with comorbidities, who constitute the majority of patients seeking psychiatric treatment, are excluded from clinical trials. Hence, there is no information about their response in the controlled studies that form the basis of evidencebased medicine, which in turn is used to establish guidelines. In other words, clinical trials conducted with oranges, ie, patients without psychiatric comorbidities, are used to produce guidelines for apples, ie, patients with psychiatric comorbidities.

Furthermore, the patients invited to participate in the typical clinical trial do not present medical comorbidities. However, medical comorbidities are very common in psychiatric patients. For example, a large epidemiological study using data from 3 German statutory health insurers during the period 2005-2007 showed that $99.8 \%$ of "severe depressive patients" 
(defined as ICD-10 F32.2, F32.3, F33.2 and F33.3; $\mathrm{n}=110,462)$ also presented a "somatic comorbidity." We also have no information about their response in controlled studies, so there is no evidence-based medicine for them.

It seems pretty obvious that we have a problem here. We are using the wrench that we have at hand, but it does not fit the nut well. I think that the evidence must be interpreted and not used blindly, because it is often based on a different group from that to which it is applied.

Another question is who creates the evidence? Evidence is based on clinical trials. So, who conducts clinical trials? Sometimes universities conduct such trials, but much more often they are carried out by pharmaceutical companies. Pharmaceutical companies will conduct clinical trials on medicines that are protected by patent rights. Besides having the financial resources, they have both the motivation and necessity to perform such trials. Therefore, there is asymmetry in the number of clinical trials of new and old medications. Could that lead to a false perception of the efficacy or utility of a medication? Yes, of course, and a seminal example of this situation was published by Goodwin et al. ${ }^{3}$ They published a large retrospective study including a population-based sample of 20,638 bipolar patients treated with lithium, divalproex, or carbamazepine. At that time, a considerable number of papers about the efficacy of divalproex had been published, and there was a shift in prescription preference from lithium to divalproex. The lithium/divalproex ratio for the first prescription shifted from approximately 6:1 in 1994 to 1:2 in 2001. The question is, was this evidence-based behavior useful? Did it lead to improved health and wellbeing for the patients? At least in relation to one very important outcome, attempted-suicide or death by suicide, it was a bad shift. The number of suicide attempts, suicides attempts requiring hospitalization, and deaths by suicide was much higher in patients using divalproex than lithium. This shift in prescription behavior came about due to the number of clinical trials conducted with divalproex, a drug that was, at that time, protected by patent rights. This directed research effort contrasted with the alternative drug, lithium, an orphan, which was not protected by patent rights. The ultimate question here is, are we using evidence-based medicine or patent-based medicine?

Finally, for practical reasons, I would like to propose the concept of the congruency and incongruency of a treatment. What is a congruent treatment? A simple example is a patient complaining of obesity, diabetes, and arterial hypertension. A congruent treatment is a correct diet. In other words, it is a treatment that will resolve or ameliorate the 3 conditions, or to use a term from evidence-based medicine, when the evidence is the same for the 3 comorbidities.
In psychiatry, a good example would be a patient suffering from generalized anxiety and depression, a pretty common comorbidity. ${ }^{4}$ In this case the patient could be treated with an SSRI, for example paroxetine, which is effective in the treatment of both disorders. ${ }^{5}$ The evidence is congruent, because it is the same for the 2 disorders.

However, this is not the most common situation. It is more common to see patients with 2 associated comorbid disorders for which the evidence suggests treatments that are not only different, but incongruent. In others words, the treatment proposed by evidence-based medicine for one could worsen the other. For example, it was found that $13.1 \%$ of patients with obsessive-compulsive disorder also present type I or II bipolar disorder. ${ }^{6}$ The evidence-based treatment proposed for OCD patients is high doses of SSRIs ${ }^{7}$ antidepressants that could worsen bipolar disorder, leading to an acceleration in cycling of this disorder, ${ }^{8}$ which in severe cases might impede also any improvement in the OCD symptoms. Seen from the other side, the use of some evidenced-based treatments for bipolar disorder, such as quetiapine ${ }^{9}$ or olanzapine, ${ }^{10}$ may increase OCD symptoms.

In such a situation, not only are the guidelines useless, but their application is also deleterious. What can we do in this case? For the $0.33 \%$ of the population $(2.5 \%$ of the population has OCD, $13.1 \%$ of which is about $0.33 \%$ ) who has this very harmful comorbidity, there is no guideline! In terms of the population of the US, it means about 1 million people have obsessive-compulsive disorder and bipolar disorder comorbidity, which would correspond to 24 million people in the world. There are some options that could help people who have this comorbidity. The solution is to identify which treatments would be effective in both conditions. At least 2 medications that could be used to treat OCD could also improve, or at least not worsen, bipolar symptoms: aripiprazole $^{11}$ and amisulpride. ${ }^{12}$

How common are these incongruent comorbidities? In fact, they are very common. For example, a 10-year prospective study found that anxiety disorder was comorbid in $53 \%$ of patients suffering from subthreshold bipolar disorder, $55.4 \%$ of patients with bipolar I disorder, and $59 \%$ of patients with bipolar II disorder. ${ }^{1}$ For all the anxiety disorders included (generalized anxiety disorder, panic disorder, agoraphobia without panic disorder, social phobia, simple phobia, posttraumatic stress disorder, and obsessive compulsive disorder), the recommended evidenced-based pharmacological treatments include serotonergic antidepressants that could trigger switches in mania and worsen the prognosis of bipolar disorder. In others words, we do not have evidence-based treatments for a comorbid situation that affects $50 \%$ of patients with bipolar disorder.

These patients, for whom there is no evidence and therefore no guidelines, not only exist, but they are very common! 
To conclude, guidelines are developed for 2 basic reasons: to help clinicians choose the best treatment and to ensure a homogeneous training of a scientifically based practice (indeed perhaps a minimal homogeneous training). However, these aims are not being achieved because, as shown above, the guidelines developed so far are not applicable to the majority of real-life patients. Hence, the guidelines, as they are, should not be followed automatically.

On the other hand, the development of more complex guidelines that reflect the epidemiological reality would be very useful. Psychiatrists and patients would benefit from having guidelines for at least the most frequent comorbidities.

Furthermore, the fact that most patients have comorbidities reflects the inconsistency of our psychiatric nosological system. This is an opportunity for research not only into nosology but also into neurobiology. Progress in this area would allow the development of a more valid diagnostic system and provide an understanding of the underlying neurobiological causes, thus leading to the development of more effective treatments.

\section{Disclosures}

Dr. Schmidt do Prado Lima reports speeches for Lundbeck in the last 12 months.

\section{REFERENCES:}

1. Zimmermann P, Brückl T, Nocon A, et al. Heterogeneity of DSM-IV major depressive disorder as a consequence of subthreshold bipolarity. Arch Gen Psychiatry. 2009; 66(12): 1341-1352.
2. Gaebel W, Kowitz S, Fritze J, et al. Uses of health care services by people with mental illness: secondary data from three statutory health insurers and the German Statutory Pension Insurance Scheme. Dtsch Arztebl Int. 2013; 110(47): 799-808.

3. Goodwin F, Fireman B, Simon GE, Hunkeler EM, Lee J, Revicki D. Suicide risk in bipolar disorder during treatment with lithium and divalproex. JAMA. 2003; 290(11): 1467-1473.

4. Kessler RC, DuPont RL, Berglund P, Wittchen HU. Impairment in pure and comorbid generalized anxiety disorder and major depression at 12 months in two national surveys. Am J Psychiatry. 1999; 156(12): 1915-1923.

5. Rickels K, Zaninelli R, McCafferty J, Bellew K, Iyengar M, Sheehan D. Paroxetine treatment of generalized anxiety disorder: a double-blind, placebo-controlled study. Am J Psychiatry. 2003; 160 (4): 749-756.

6. Timpano KR, Rubenstein LM, Murphy DL. Phenomenological features and clinical impact of affective disorders in OCD: a focus on bipolar disorder and OCD connection. Depress Anxiety. 2012; 29(3): 226-233.

7. Taylor D, Paton C, Kapur S. Anxiety Spectrum Disorders. In: Taylor D, Paton C, Kapur S. The Maudsley Prescribing Guidelines. 10th ed. London, UK: Informa Healthcare; 2009: 234-239.

8. Goodwin FK, Jamison KR. Medical treatment of depression. In: Goodwin FK, Jamison KR. Manic-Depressive Illness. 2nd ed. Oxford, UK: Oxford University Press; 2007: 747-796.

9. Stamouli S, Lykouras L. Quetiapine-induced obsessive-compulsive symptoms: a series of five cases. J Clin Psychopharmacol. 2006; 26(4): 396-400.

10. Mottard JP, de la Sablonnière JF. Olanzapine-induced obsessive-compulsive disorder. Am J Psychiatry. 1999; 156(5): 799-800.

11. Sayyah M, Sayyah M, Boostani H, Ghaffari SM, Hoseini A. Effects of aripiprazole augmentation in treatment-resistant obsessivecompulsive disorder (a double-blind clinical trial). Depress Anxiety. 2012; 29(10): 850-854.

12. Metin O, Yazici K, Tot S, Yazici AE. Amisulpride augmentation in treatment resistant obsessive-compulsive disorder: an open trail. Hum Psychopharmacol. 2003; 18(6): 463-467. 